\section{Still un-sirtuin}

\section{By Lev Osherovich, Senior Writer}

Researchers at the NIH have proposed a new mechanism to explain the beneficial metabolic effects of resveratrol, a polyphenol compound from red wine thought by many to act primarily on sirtuin 1 . The NIH team suggests resveratrol works by inhibiting phosphodiesterase-4, an enzyme that sits far upstream of sirtuin 1 and affects a signaling pathway that leads to increased energy utilization. ${ }^{1}$

The findings could provide repurposing opportunities for the plethora of phosphodiesterase-4 (PDE-4) inhibitors on the market or in the clinic, primarily for pulmonary indications.

Prior work by researchers at Harvard University and the Massachusetts Institute of Technology suggested resveratrol promotes

Figure 1. Explaining resveratrol's metabolic effects. Park et al. propose that the principal target of resveratrol is phosphodiesterase 4 (PDE-4), not sirtuin 1 (SIRT1) as previously thought.

The team found that resveratrol can inhibit PDE-4, an enzyme that degrades cyclic AMP (CAMP) in response to signaling by glucagon.

Glucagon is a peptide hormone that stimulates metabolic activity. Normally, extracellular glucagon works through glucagon receptor (GCGR) [a] to stimulate adenylate cyclase 1 (ADCY1; AC1) to convert ATP into CAMP, which in turn stimulates rap guanine nucleotide exchange factor 3 (RAPGEF3; EPAC) [b]. Higher EPAC activity indirectly leads to activation of AMP-activated protein kinase (AMPK) [c], a key regulator of cellular energy utilization.

Park et al. report that resveratrol inhibited PDE-4 in vitro and stimulated EPAC activity and AMPK phosphorylation in cell culture [d]. In a mouse model of diet-induced obesity, the generic PDE-4 inhibitor rolipram increased AMPK activity [e] and SIRT1 activation [f]. Based on these observations, Park et al. suggest the metabolic effects of resveratrol are likely due to PDE-4 inhibition.

At least 16 PDE-4 inhibitors are marketed or in development for a range of inflammatory and pulmonary indications, including Daliresp roflumilast from Takeda Pharmaceutical Co. Ltd., Mitsubishi Tanabe Pharma Corp. and Forest Laboratories Inc., which is marketed to treat chronic obstructive pulmonary disease (COPD), the consumption of energy and other antidiabetic effects by activating sirtuin 1 (SIRT1), a protein deacetylase implicated in a variety of metabolic and neurodegenerative processes. ${ }^{2}$ Indeed, that research led to the formation of Sirtris Pharmaceuticals Inc., which was acquired in 2008 by GlaxoSmithKline plc for $\$ 651$ million.

In 2010, GSK discontinued development of Sirtris' lead compound, SRT501, after data from an open-label Phase IIa trial in 24 patients showed the orally bioavailable formulation of resveratrol had minimal efficacy and increased the risk of renal complications in patients with multiple myeloma (MM).

The pharma's next-generation SIRT1 activator, SRT2104, has completed Phase II testing for type 2 diabetes and is in Phase I testing for cardiovascular and inflammatory indications. GSK has at least two other SIRT1 activators in Phase I testing in inflammatory indications.

While the molecules were moving through the clinic, independent teams at several academic institutions and at Elixir Pharmaceuticals Inc., Amgen Inc. and Pfizer Inc. reported that, in their hands, resveratrol and three other Sirtris compounds, not including SRT2104, did not directly activate SIRT1 in vitro. ${ }^{3-6}$

"The question is how can these compounds activate SIRT1 if they

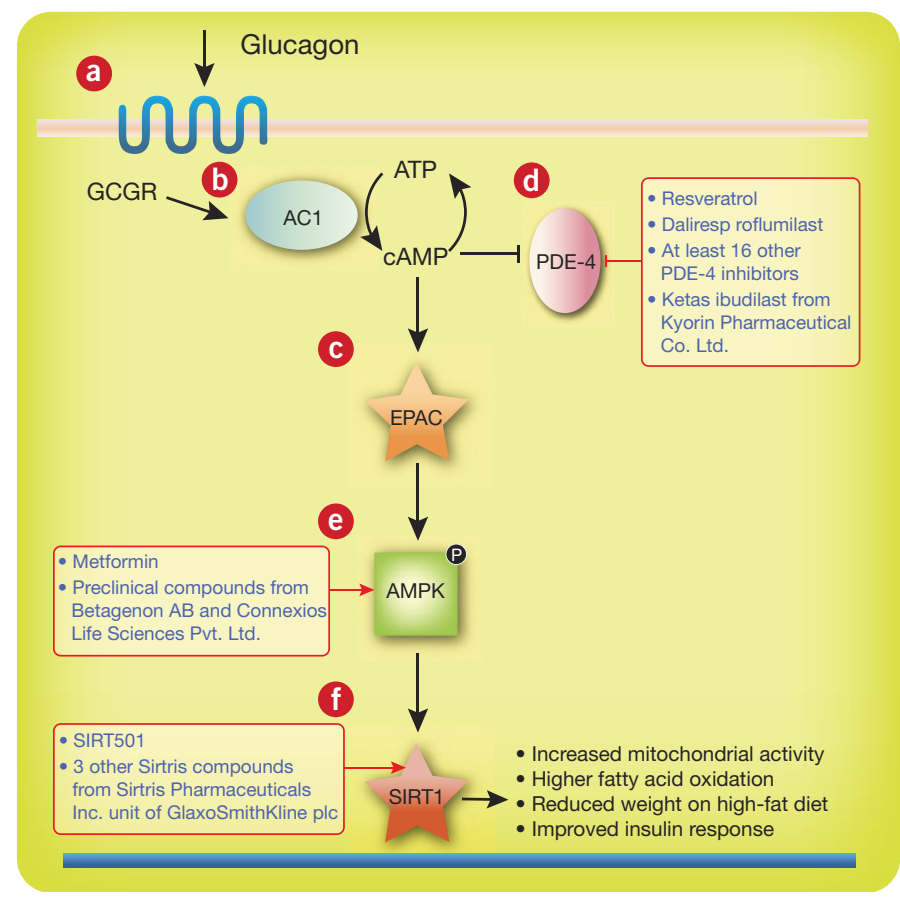
and Ketas ibudilast from Kyorin Pharmaceutical Co. Ltd., which is marketed in Japan to treat asthma and stroke.

Betagenon AB and Connexios Life Sciences Pvt. Ltd. have AMPK activators in preclinical development for type 2 diabetes and obesity. AMPK also is the principal target of metformin, a generic drug to treat type 2 diabetes.

In 2010, GlaxoSmithKline plc discontinued development of SRT501, an orally bioavailable formulation of resveratrol, after data from an open-label Phase Ila trial in 24 patients showed the compound had minimal efficacy and the potential to indirectly exacerbate renal complications in patients with multiple myeloma (MM).

The pharma's SIRT1 activator SRT2104 has completed Phase II testing for type 2 diabetes and is in Phase I testing for various cardiovascular and inflammatory indications. GSK has at least two other SIRT1 activators in Phase I testing. 


\section{ANALYSIS}

\section{TARGETS \& MECHANISMS}

don't directly activate it," said Jay Chung, chief of the Laboratory of Obesity and Aging Research at the NIH's National Heart, Lung, and Blood Institute.

\section{"We believe that these compounds indirectly activate SIRT1 by inhibiting phosphodiesterases. We have identified the pathway by which cAMP can confer metabolic benefits, including SIRT1 activation." - Jay Chung, \\ National Heart, Lung, and Blood Institute}

New findings from Chung's group point to PDE-4 as a potential target of resveratrol. "Our findings elucidate one possible mechanism for resveratrol's action," said Chung.

\section{To PDE or not to PDE?}

Chung's team examined the metabolic effects of resveratrol in mice and in cultured mammalian cells. Compared with vehicle, resveratrol raised levels of cyclic AMP (cAMP), a signaling molecule that activates metabolic pathways in response to signaling by glucagon. Glucagon is a peptide hormone that promotes energy utilization.

Building on previous studies by other teams suggesting that resveratrol can inhibit phosphodiesterases, ${ }^{7}$ a class of cAMP-degrading enzymes, Chung hypothesized that resveratrol inhibition of phosphodiesterases could account for the compound's effect on cAMP.

To test the idea, the group screened a panel of phosphodiesterases and found resveratrol potently blocked PDE-4.There are two marketed PDE-4 inhibitors: Daliresp roflumilast, a selective compound from Takeda Pharmaceutical Co. Ltd., Mitsubishi Tanabe Pharma Corp. and Forest Laboratories Inc. marketed to treat chronic obstructive pulmonary disease (COPD), and Ketas ibudilast, a nonselective compound marketed in Japan by Kyorin Pharmaceutical Co. Ltd. to treat asthma and stroke. At least 16 other PDE-4 inhibitors are in development.

Chung's team worked its way down the pathway and showed that resveratrol could turn on AMP-activated protein kinase (AMPK), a central metabolic regulator that in turn activates SIRT1 (see Figure 1, "Explaining resveratrol's metabolic effects").

In cell culture, a generic PDE-4 inhibitor increased cAMP levels, AMPK signaling and SIRT1 activity compared with vehicle. In a mouse model of diet-induced obesity, the PDE-4 inhibitor modestly decreased body fat and glucose tolerance compared with vehicle.

Results were reported in Cell and are unpatented.

"We believe that these compounds indirectly activate SIRT1 by inhibiting phosphodiesterases," said Chung. "We have identified the pathway by which cAMP can confer metabolic benefits, including SIRT1 activation."

George Vlasuk, CEO of GSK's Sirtris unit, told SciBX he thinks Chung's conclusions are based on circumstantial evidence.

Chung's team "investigated the effects of a selective PDE-4 inhibitor in a mouse model of diet-induced obesity and proceeded to circumstantially link the beneficial effects of this compound in this setting to the inhibition of phosphodiesterase activity by resveratrol," said Vlasuk.

He thinks that because Chung's team did not do a direct comparison of the two compounds in the same models, "one cannot link the observed metabolic benefits of both PDE-4 inhibition and resveratrol."
Chung said that because resveratrol interacts with more phosphodiesterases than just PDE-4, he would not expect PDE-4 inhibition to exactly match resveratrol's effects. He added that the data in the paper show that, at least qualitatively, PDE-4 inhibition reproduces the overall effects of treatment with resveratrol.

Vlasuk also said the plasma concentration needed to achieve beneficial effects with resveratrol is much lower than the concentration of resveratrol needed to inhibit PDE-4 in vitro. Thus, he suspects that if resveratrol does indeed inhibit phosphodiesterases, this activity does not account for its metabolic effects.

Matt Kaeberlein, associate professor of pathology at the University of Washington, is on the fence about Chung's hypothesis. Kaeberlein has previously published evidence against resveratrol's direct activation of SIRT1.4 "People have speculated that some aspects of resveratrol's activity are mediated through AMPK, whether directly or not," he said.

Kaeberlein added that Chung's findings "fit with the idea that there may be a target for resveratrol upstream of AMPK, but I'm skeptical that PDE-4 accounts for all the effects of resveratrol."

Indeed, because resveratrol is a fairly promiscuous molecule that binds to multiple targets, "we cannot prove that there is no other mechanism," said Chung.

\section{Additive or synergistic}

One way to test Chung's hypothesis would be to combine resveratrol and a PDE-4 inhibitor to see whether the two compounds have an additive effect, as would be expected for a common target, or a synergistic effect, as would be expected for separate targets.

Chung told SciBX that such an experiment could be informative.

Ultimately, Chung said, the promiscuous nature of resveratrol and the complex regulation of metabolism make it hard to nail down how the molecule really works. "It is not clear whether there is a definitive experiment that can resolve this question to everyone's satisfaction," he said.

Kaeberlein agreed, noting that a different approach to the question of resveratrol's target could come from experiences in the clinic. $\mathrm{He}$ suggested that Chung's team "look in patients who are already being treated with PDE-4 inhibitors to see if there's protection against diabetes."

Chung may soon get such data. He plans to conduct a clinical trial of an undisclosed PDE-4 inhibitor in obesity and metabolic syndrome in partnership with an undisclosed pharma company.

Osherovich, L. SciBX 5(7); doi:10.1038/scibx.2012.170

Published online Feb. 16, 2012

\section{REFERENCES}

1. Park, S.-J. et al. Cell; published online Feb. 3, 2012; doi:10.1016/j.cell.2012.01.017

Contact: Jay H. Chung, National Institutes of Health, Bethesda, Md.

e-mail: chungj@nhlbi.nih.gov

2. Baur, J.A. \& Sinclair D.A. Nat. Rev. Drug Discov. 5, 493-506 (2006)

3. Borra, M.T. et al. J. Biol. Chem. 280, 17187-17195 (2005)

4. Kaeberlein, M. et al. J. Biol. Chem. 280, 17038-17045 (2005)

5. Beher, D. et al. Chem. Biol. Drug Des. 74, 619-624 (2009)

6. Pacholec M. et al. J. Biol. Chem. 285, 8340-8351 (2010)

7. Dell'Agli, M. et al. J. Agric. Food Chem. 53, 1960-1965 (2005) 


\section{ANALYSIS}

COMPANIES AND INSTITUTIONS MENTIONED

Amgen Inc. (NASDAQ:AMGN), Thousand Oaks, Calif.

Elixir Pharmaceuticals Inc., Cambridge, Mass.

Forest Laboratories Inc. (NYSE:FRX), New York, N.Y.

GlaxoSmithKline plc (LSE:GSK; NYSE:GSK), London, U.K.

Harvard University, Cambridge, Mass.

Kyorin Pharmaceutical Co. Ltd. (Tokyo:4569), Tokyo, Japan

Massachusetts Institute of Technology, Cambridge, Mass.

\section{TARGETS \& MECHANISMS}

Mitsubishi Tanabe Pharma Corp. (Tokyo:4508; Osaka:4508), Osaka, Japan

National Heart, Lung, and Blood Institute, Bethesda, Md.

National Institutes of Health, Bethesda, Md.

Pfizer Inc. (NYSE:PFE), New York, N.Y.

Sirtris Pharmaceuticals Inc. (NASDAQ:SIRT), Cambridge, Mass. Takeda Pharmaceutical Co. Ltd. (Tokyo:4502), Osaka, Japan

University of Washington, Seattle, Wash. 